\title{
AfricArXiv
}

\section{Call to action: COVID-19 Rapid Review}

\section{AfricArXiv}

Published on: Sep 22, 2020

DOI: $10.21428 / 3 b 2160 c d .0 b 9 e 08 b f$

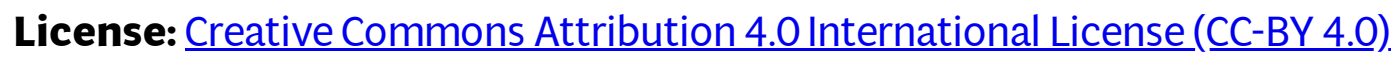




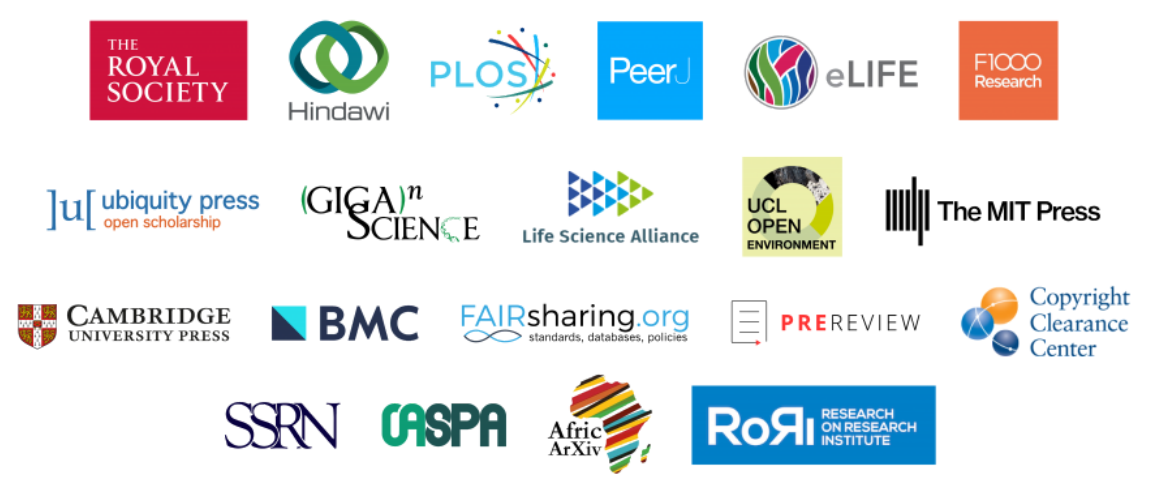

As a supporter and signatory of the COVID-19 Publishers Open Letter of Intent for Rapid Review we call upon researchers in Africa and beyond to join the initiative and take action in one or more of the following ways

- Submit your COVID-19 related manuscript via AfricArXiv to one of our partner repositories at https://info.africarxiv.org/submit/

- suggest a preprint for peer review on https://outbreaksci.prereview.org/

- Sign sign up in this form as a volunteer reviewer with suitable expertise relevant to COVID-19 from any career stage and discipline to sign up to a "rapid reviewer pool" and commit to rapid reviewing times, along with an upfront agreement that your reviews and identity can be shared among publishers and journals if submissions get rerouted.

To discover digitally archived preprint manuscripts with a focus on C19 research from and about Africa, please refer to the following collections:

- AfricArXiv/ScienceOpen collection https://www.scienceopen.com/collection/COVID19_Africa $>>$ (preprints only)

- AAS Open Research collection https://aasopenresearch.org/collections/coronavirus

- medRxiv AND bioRxiv (search settings abstract/title and COVID-19 or SARS-CoV-2 or coronavirus in title) 


\section{For context}

On 27 April 2020, a group of publishers and scholarly communications organisations announced a joint initiative to maximize the efficiency of peer review, ensuring that key work related to COVID-19 is reviewed and published as quickly and openly as possible. OASPA fully supports this collaborative approach and is pleased to host the Open Letter of Intent below.

\section{April 2020 (updated 11 August 2020)}

The COVID-19 pandemic has created a new urgency to openly and rapidly share and review COVID-19 research.

We, a group of publishers and scholarly communications organizations, are committing to work together on a cross-publisher rapid review and review transfer initiative. With the endorsement of the Open Access Scholarly Publishers Association (OASPA) we are making the following calls to reviewers, editors, authors, and publishers in the research community, in order to maximize the efficiency and speed of the triage and peer review process of COVID-19 research.

To reviewers and authors:

1. We call on volunteer reviewers with suitable expertise relevant to COVID-19 from all career stages and disciplines, including those from industry, to sign up to a "rapid reviewer pool" and commit to rapid reviewing times, along with an upfront agreement that their reviews and identity can be shared among publishers and journals if submissions get rerouted. Please sign up in this form.

2. We call on volunteer reviewers (whether or not they have signed up for rapid review) to identify and highlight important and crucial COVID-19 preprints (e.g. by using_ https://outbreaksci.prereview.org(), as early as possible, to optimise the limited time of expert reviewers who are subsequently invited to review the most important and promising research by a journal/platform.

3. We call on authors to support reviewers and publishers in this endeavour by ensuring the deposition of their submission as a preprint, and by working with publishers to make the peer-reviewed article and associated dataset, software, and model available for reuse as rapidly as possible. 
To publishers and editors:

1. We call on all publishers to actively facilitate posting of COVID-19 preprints to preprint servers with the agreement of the authors, if authors have not already posted a preprint. This should be after confirming the submission warrants further review. (It is understood preprint servers are also doing their own checks and

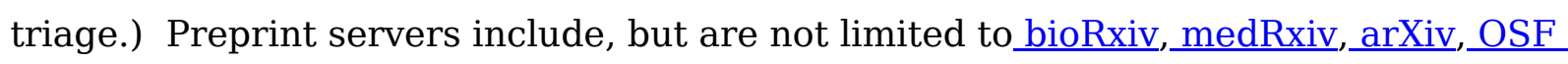
Preprints, SciELO Preprints, SSRN etc, depending on research scope.

2. We call on all publishers and editors to consider comments on preprints during the journal peer-review process.

3. We call on all publishers to ensure all COVID-19 submissions include a mandatory data availability statement, if they do not already do this for all submissions.

- Publishers should aim to facilitate the stewardship of FAIR data and software code sharing underlying prioritised COVID-19 papers (and associated preprints) during the pandemic by working with_FAIRsharing, the Research Data Alliance and Force11 via the joint RDA/Force11 FAIRsharing Working_Group (e.g. providing recommendations to appropriate repositories and use of relevant data and metadata standards).

This call is in addition to supporting these Wellcome Trust-coordinated calls: "Sharing_ research data and findings relevant to the novel coronavirus (COVID-19) outbreak" and "Publishers make coronavirus (COVID-19) content freely available and reusable".

Signatories:

eLife, F1000 Research, Hindawi, PeerJ, PLOS, Royal Society, FAIRsharing, Outbreak Science Rapid PREreview, GigaScience, Life Science Alliance, Ubiquity Press, UCL, MIT Press, Cambridge University Press, BMC, RoRi and AfricArXiv

Downloadable PDF of this Letter of Intent (updated August 2020). 